\title{
Effect of PaCket Size on TCP-Reno Performance OVER LOSSY, CONGESTED LINKS
}

\author{
Marcelo Albuquerque ${ }^{\diamond}$, Jae H. Kim ${ }^{\diamond}$, Scnior Mcmbcr, IEEE, and Sumit Roy ${ }^{\star}$, Senior Member, IEEE \\ $\diamond$ Phantom Works of The Boeing Company, Seattle, WA \\ * Department of Electrical Engineering, University of Washington, Scattlc, W $\Lambda$
}

\begin{abstract}
This paper investigates the performance of a single TCP-Reno connection over a lossy, congested link as a function of TCP packet size on the forward link and the ratio $\alpha$ of forward-to-reverse link capacity measured in terms of packets/sec. $\alpha>1$ constitutes the primary scenario of interest since the reverse link (for the $A C K s$ ) is a bottleneck, which directly impacts the forward link throughput. Congestion at the forward buffer is induced by assuming buffer size to be significantly less than the bandwidth-delay product of the round-trip connection. Further, forward link error characteristics are modeled as a continuous-time two-state Markov chain, where packets are either transmitted free of errors (good state) or lost (bad state). By tuning the model parameters, the link error process can be made to vary from independent loss to (highly) correlated loss scenarios. Results show that TCP-Reno performance benefits from larger packet sizes for connections dominated by link errors; throughput is less sensitive to packet size when the connection is dominated by forward path congestion. Additionally, performance sensitivity to variation of forward buffer size and the round-trip propagation delay is discussed and supported by simulation results using ns-2 Network Simulator.
\end{abstract}

Keywords - TCP-Reno, asymmetry, correlated errors, performance analysis

\section{INTRODUCTION}

TCP performance over an end-to-end path that includes a wireless/satellite segment is a function of several important parameters: link loss characteristics and tuning of various TCP parameters such as buffer and packet size (which depend on the channel error). Further, in TCP over satellite, applications typically suffer from channel asymmetry, where the forward link capacity (measured in packets/sec) greatly exceeds that of the reverse link, leading to further underutilization of the forward link. In this work, we focus specifically on the choice of TCP packet size that fundamentally affects TCP dynamics since the sender's current congestion window $W(t)$ is adjusted in units of packets depending on receipt (or non-receipt) of acknowledgements (ACKs). Thus when $W(t)$ is incremented by 1 packet, the equivalent increase in throughput (measured in bits/sec) is greater for con- nections using larger packet sizes. We investigate the issue of optimal packet sizing in the presence of $t w o$ types of packet losses - due to i) buffer overflow (congestion loss) and ii) channel loss, respectively. It is useful to characterize links dominated by congestion losses as presenting a "busy connection", while those dominated by link errors as presenting a "lossy connection".

\section{TCP BACKGROUND}

\section{A. Congestion Control Algorithm}

A TCP sender transmits data packets ${ }^{1}$ in sequence and expects to receive acknowledgements (ACKs) confirming their successful reception. The maximum number of packets allowed to be outstanding in the network at time $t$ is known as the congestion window size $W(t) . W(t)$ is typically maintained in units of bytes but for convenience it is expressed in units of packets here, unless otherwise noted. ACKs generated by the standard TCP receivers carry the information about the last in-order data packet received; out-of-order packets are stored but not acknowledged until all previously transmitted packets have been received. TCP uses this continuous flow of ACKs as a way of probing the network and detecting signs of congestion. It adapts itself by dynamically adjusting $W(t)$ in one of two distinct phases, known as slowstart and congestion avoidance phases. TCP switches from slow-start to congestion avoidance when $W(t)$ reaches the slow-start threshold $W_{\text {ssth }}(t)$, that is also dynamically adjusted. The slow start phase allows for an exponential increase of $W(t)$ that grows up to $W_{\text {ssth }}(t)$ by incremets of one (packet) for every "new" $\mathrm{ACK}^{2}$ received. On the other hand, congestion avoidance phase allows for a linear window growth with time by incrementing $W(t)$ by 1 for every (current) window's worth of new ACKs received.

\footnotetext{
${ }^{1}$ Although the term "segment" is widely used in the literature we use "packet" throughout this paper.

${ }^{2}$ We use "new" ACK to refer to those acknowledging data not previously acknowledged; "repeated" ACKs acknowledge previously ACKed packets.
} 
The sender deduces the congestion state of the network by two different means - in the first, the time elapsed between the transmission of a data packet and the reception of its corresponding ACK, known as the round-trip time ( $R T T$ ) is compared to an adaptive threshold, known as the retransmission time-out ( $R T O$ ); if $R T T$ exceeds $R T O$, the sender assumes the packet has been lost. The second technique requires the sender to monitor the number of consecutive repeated ACKs received (that signifies a break of insequence received packets); if it reaches a threshold $K$ (usually $K=3$ ), a congestion loss is assumed. The following summarizes the congestion control mechanism actions taken by TCP-Reno in the event of packet. loss:

1) Time-Out

- Set $W(t+)=1$ and $W_{s s t h}(t+)=W(t) / 2$.

- Retransmit the lost packet and resume slow-start phase.

2) $K$ Repeated ACKs

- Fast-retransmit the lost packet.

- Set $W(t+)=W(t) / 2+K$ and $W_{s s t h}(t+)=W(t) / 2$

- While a new ACK is not received, increment $W(t+)$ by one for every repeated ACK and transmit new packets if possible (Fast-Recovery Phase).

- Upon receiving a new ACK, set $W(t+)=W(t) / 2$ and resume congestion avoidance phase.

While in fast-recovery phase, assuming each repeated ACK indicates a packet has been successfully received allows the sender to avoid dropping the number of outstanding packets in the network below $W(t) / 2$. If that happens, the result will be a transmission burst upon reception of the first new ACK.

\section{B. Effects of Asymmetry}

Specific problems arise when TCP is used over asymmetric connections, sincc TCP rclics on a regular flow of ACKs on the reverse path to advance its forward congestion window. When $\alpha>1$ (reverse link is the bottleneck), it results in further under-utilization of forward link capacity. As $W(t)$ grows, the reverse link becomes fully utilized and reverse buffer overflow follows. As $W(t)$ is further increased, the forward link becomes fully utilized at which point only 1 out of $\alpha$ ACKs "survives" the reverse buffer. Finally the forward buffer overflows triggering TCP's congestion avoidance mechanisms. Previous studics on the effects of link asymmetry on TCP performance highlight the following three observations:

1. Increased burstiness on forward transmissions due to ACK losses in the reverse link - the "surviving"
ACKs acknowledge multiple packets and as a result the sender releases multiple new packets into the forward buffer.

2. Slower congestion window growth since only a fraction of ACKs generated by the receiver actually reach the sender and the fact that the TCP window evolution algorithm is based on number of ACKs received and not the amount of data acknowledged.

3. Reduced effectiveness of the fast-retransmit algorithm due to ACK loss, since it depends on the reception of at least 3 ACKs to be triggered. Besides, ACKs for retransmission may be lost resulting in timeouts.

A pioneering analysis of the fundamental issues involving link asymmetry was carried out in [1] where it was shown that there are three modes of operation based on the relationship between the normalized asymmetry factor $\alpha$ (defined as the ratio of time to transmit an ACK in the reverse link to that of a data packet in the forward link) and the forward buffer size $B_{f}$. When $\alpha>1$ (the usual scenario of interest), for $B_{f}<\alpha$, the forward buffer size is insufficient for the bursts of $\alpha$ packets that will occur when the forward link is fully utilized. For $B_{f}>3 \alpha$, the forward buffer will still not be cmpty by the time the sender receives 3 repeated $A C K s$ and detects a packet loss due to congestion in the forward link. As a result, the retransmission will find the forward link fully utilized, its corresponding $\Lambda \mathrm{CK}$ will find the reverse buffer full, and it is likely to be dropped. Thus, "normal" operation takcs place for $\alpha<B_{f}<3 \alpha$, when the above effects are not seen. An initial approach to solving the link asymmetry problem was to reduce $\alpha$ by using smaller ACKs. It was proposed in [2], [3] and [4] to comprcss TCP/IP headers by exploiting the redundancy between subsequent packets. Another solution early proposed was the use of delayed-ACKs - due to the cumulative nature of ACKs in TCP, the receiver may avoid overflowing the reverse buffer by generating less than one ACK for every data packet received. In [5], an optimal dclayed- $\Lambda$ CK mcchanism is described. Its use alleviates congestion in the reverse link but does not address the problems of forward transmission burstiness and slow congestion window growth. In [6] a solution involving modifications only to the TCP stack at the receiver's terminals is proposed in ordcr to dynamically adjust the number of packets acknowledged per ACK (ppa). In [7], the author evaluates the usc of some important algorithms but does so for a connection where the reverse link does not reprcsent the bottleneck. A more complete and detailed 
analysis of the effects of link and latency asymmetry is presented in [8]; several techniques are suggested to address all three problems that arise with link asymmetry listed above.

\section{Effects of Transmission Errors}

The control action mechanism in TCP scnders was designed to respond to congestion (buffer overflow) losses. Any packet loss in the forward link duc to other causes such as link errors are therefore interpreted by the sender as arising duc to network congestion, leading to reduction of the transmission rate, thereby needless under-utilizing network resourccs. Wc notc that transmission errors in the forward link impact TCP connection throughput far more than errors on the reverse channel, since ACKs in TCP are cumulative. Thus whenever an ACK is lost, it's information is resident in all subsequent ACKs. Nonetheless, ACK losses still impact forward TCP throughput in a similar manner as reverse buffer overflows do in asymmetric connections.

\section{System MODEL}

We assume a persistent data source (e.g. an ftp application) operating over forward and reverse paths with capacity $\mu_{f}$ and $\mu_{r}$ packets/second and FIFO forward and reverse buffers of sizes $B_{f}$ and $B_{r}$, respectively. The resulting normalized asymmetry factor is $\alpha=\mu_{f} / \mu_{r}$ [1], and normalized forward buffer size $\beta=B_{f} / \mu_{f} \tau$ where $\tau$ is the total (round-trip) propagation delay. The forward link drops data packets due to link-layer errors based on a continuous-time, two-state model described next.

The channel alternates between two states - the "good" and the "bad" denoting the extent of packet loss. It remains in the present state for an amount of time defined as an exponential random variable with average $\tau_{g}\left(\tau_{b}\right)$, before switching to the other state. The probability of a packet being lost due to error in good(bad) state is $p_{g}\left(p_{b}\right)$. For simplicity, we assume $p_{g}=0$ and $p_{b}=1$ in our work. 'I'he resultant packet loss process is a hidden Markov model with the important feature that it is not independent, but correlated in general. The channel state transitions drive TCP's congestion control algorithm; thus the sequence of packet losses experienced by TCP sender is a result of the interaction between TCP's own feedback mechanism (congestion control) and the channel state evolution. Consequently it is impossible to translate channel statistics to that of the actual packet loss events and throughput results will be presented in terms of a channel "unavailability" factor $\rho=\tau_{b} /\left(\tau_{g}+\tau_{b}\right)$. It is straightforward to verify that correlation between error events can be increased by increasing $\tau_{b}$ (and $\tau_{g}$ correspondingly) for a fixed $\rho$.

We make the important distinction between connections whose performance are dominated by congestion losses ("busy connections") and link losses ("lossy connections". For a fixed $\rho$, increasing the correlation coefficient between errors implies larger (on average) error bursts and consequently fewer error events. Hence, for a fixed $\rho$, sufficiently large (sma.ll) values of $\tau_{b}$ indicate "busy connections" ("lossy connections"). A "lossy connection" is extremely difficult to model and will not be attempted; the saturation throughput limit in a "busy connections" will now be addressed.

\section{CONNECTIONS WITH $\alpha \leq 1$}

Though $\alpha \leq 1$ is not the primary scenario of interest, it is treated first for the result simplicity and preliminary insights it provides for $\alpha>1$.

\section{A. Saturation Throughput}

In "busy connections", reductions of window size $W(t)$ are mainly due to buffer overflows. It is expected that congestion occurs (roughly) every time $W(t)$ reaches some value $W_{\text {full }}$, which for $\alpha \leq 1$ is given by:

$$
W_{\text {full }}=\mu_{f} \tau(1+\beta)
$$

Fig.1 illustrates such behavior for two different packet sizes; $W_{\text {full }}$ (in bytes), is the samc irrcspective of the packet size being used. Note that larger packet sizes allow for faster growth of $W(t)$ and as a result cycles have smaller duration. This figure suggests identical

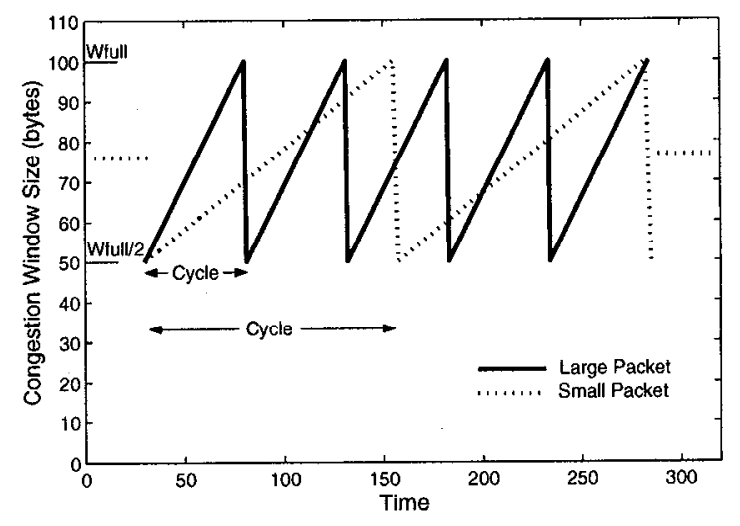

Fig. 1. Typical congestion window evolution for two different packet sizes in a "busy connection" and $\alpha \leq 1$.

throughput for both packet sizes since throughput is 
"roughly" the area under these curves. However, this estimate must be adjusted to account for time spent in the transition between cycles, defined as the time from the last transmission priori to a loss detection and the reception of the second "new" ACK subsequent to retransmission of the lost packet, which are periods of low (a.most zero) throughput. The reason for using the second "new" ACK is that in the fast-recovery algorithm, the sender must pause after retransmission while receiving $W_{\text {full }} / 2-3$ repeated ACKs; it will only then attempt a new transmission which will eventually generate a "new" ACK other than the one for the retransmission. Fig. 2 shows a closer look at the tran-

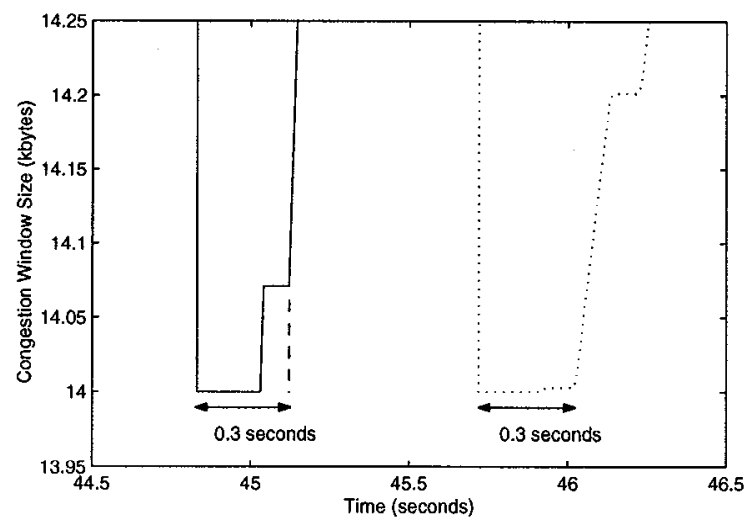

Fig. 2. Closer look at cycle transitions for 1000bytes (solid line) and 200bytes (dotted line) packets.

sition between cycles for two different packet sizes and their time duration is shown to be the same. Hence we should expect a poorer performance for larger packet sizes as the number of cycles in this case is higher.

In [9], expressions to estimate the saturation throughput are derived and details will not be presented here. The approach is to find expressions for both the time duration and the throughput of each one of two cycle stages, namely the transition phase, as described above, and the growth phase (the remaining portion of the cycle). The throughput of each cycle can then be obtained which is also the overall throughput of a "busy connection".

$T_{1}, T_{2}$ and $T_{3}$ are defined as the $R T T$ for packets transmitted in three distinct situations: (i) when a retransmission occurs (both links and buffers are fully utilized); (ii) when the sender transmits a new packet after a retransmission (the network load has been reduced by then); (iii) an average $R T T$ for packets transmitted in the growth phase. The final expression for the saturation throughput as a function of $T_{1}$, $T_{2}, T_{3}$ and $W_{\text {full }}$, normalized by the forward link ca- pacity $\mu_{f}$ is given by:

$$
\gamma_{s a t}=\frac{W_{f u l l}\left[6 T_{1}+\left(3 W_{f u l l}+2\right) T_{3}\right]}{4 T_{3} \mu_{f}\left[3 T_{1}+2 T_{2}+W_{f u l l} T_{3}\right]}
$$

Note that since $\mu_{f}$ is given in packets per second, (2) is also a function of the packet size for a fixed bandwidth.

\section{B. Simulation Results}

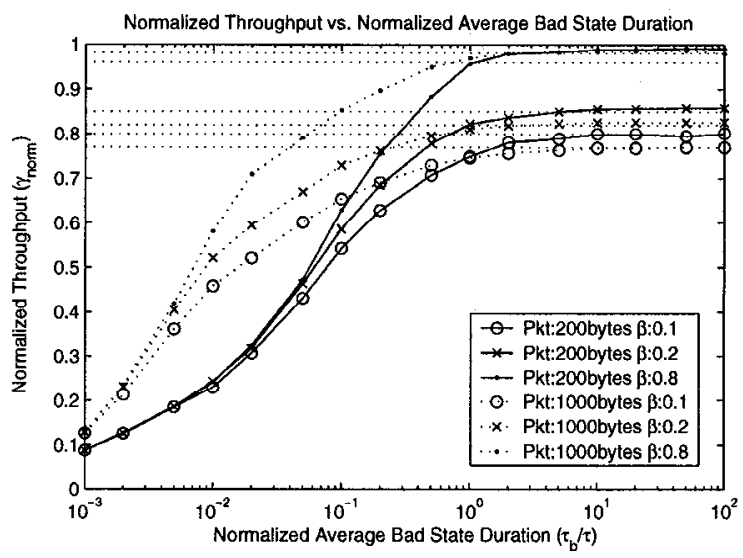

Fig. 3. Normalized throughput $\left(\gamma_{\text {norm }}\right)$ as a function of the normalized average bad state duration $\left(\tau_{b} / \tau\right)$ for $\rho=10^{-3}$. The horizontal dotted lines are results from equation (2).

We ran simulations using the Network Simulator (ns-2) [10] for various values of $\tau_{b}, \tau_{g}, \beta$ and packet size. Fig.3 shows the long-run normalized throughput $\left(\gamma_{n o r m}\right)$ as a function of $\tau_{b} / \tau$ for a fixed $\rho=10^{-3}$ and different packet sizes and values of $\beta$. The forward bandwidth is fixed at $1 \mathrm{Mbps}$ which results in $\mu_{f}=125 \mathrm{pkt} / \mathrm{s}$ and $\mu_{f}=625 \mathrm{pkt} / \mathrm{s}$ for 1000 bytes and 200 bytes packets, respectively. Other parameters are $\mu_{r}=625 \mathrm{pkt} / \mathrm{s}$ and $\tau=200 \mathrm{~ms}$. Moving from left to right in the plot, we go from lossy connections to busy connections. Smaller packet sizes yield slightly better performance for busy connections while large packet sizes allow for significantly better performance in lossy connections. The choice of $B_{f}$ is only relevant in busy connections since in lossy connections the transmission rate is never high enough to build queues in the forward buffer, specially for small packet sizes. The horizontal dotted lines in the plot represent the analytical results using (2). 'The numeric results are presented in Table I and they show that the analysis is in accordance with the simulation outcome.

\section{Connections WITH $\alpha>1$}

The more relevant case where the reverse link is a bottleneck $(\alpha>1)$ is investigated in this section. 
TABLE I

Throughput Saturation Limits for $\alpha \leq 1$.

\begin{tabular}{|c|c|c|c|}
\hline \hline$\beta$ & Pkt Size & Eq. (2) & Simulation \\
\hline \hline \multirow{2}{*}{0.1} & 200 & 0.801 & 0.802 \\
\cline { 2 - 4 } & 1000 & 0.766 & 0.770 \\
\hline \hline \multirow{2}{*}{0.2} & 200 & 0.854 & 0.859 \\
\cline { 2 - 4 } & 1000 & 0.819 & 0.826 \\
\hline \hline \multirow{2}{*}{0.8} & 200 & 0.984 & 0.991 \\
\cline { 2 - 4 } & 1000 & 0.960 & 0.979 \\
\hline
\end{tabular}

As in the previous section, an expression for the saturation throughput is presented although details are omitted.

\section{A. Saturation Throughput}

When $\alpha>1$, data packets are transmitted in the forward link in less time than it takes to transmit an ACK in the reverse link. By the time $W(t)$ reaches its maximum value $W_{\text {full }}$ and a packet is dropped, the reverse buffer will have been overflowing already, allowing at this point for only one "surviving" ACK for every $\alpha$ ACKs generated by the receiver. $W_{f u l l}$ in this case is given by:

$$
W_{f u l l}=\mu_{f} \tau(1+\beta)+\alpha B_{r}
$$

Referring again to the analysis in [9], an expression for the throughput saturation limit is derived. The results are only valid for the particular case in which only one packet is dropped at the end of a cycle and the ACK for the retransmission is dropped at the reverse buffer, causing the sender to time-out. As discussed in section II-B, the authors in [1] find that this is particularly true when $B_{f}>3 \mu_{f}$. Fig.4 illustrates a typical window evolution for this case.

The analysis in this case is much more complex and will eventually lead to less reliable results due to necessary approximations. Again three different $R T T$ estimates are obtained, $T_{4}, T_{5}$ and $T_{6}$. They correspond to the following three situations: (i) when a retransmission occurs (both links and buffers are fully utilized); (ii) an average $R T T$ for packets transmitted in slow-start phase; (iii) an average $R T T$ for packets transmitted in congestion avoidance phase. Also, an expression for $\alpha_{\text {avg }}$, defined as the average number of packets acknowledged by each "surviving" ACK during congestion avoidance, is obtained. The expression for the saturation throughput as a function of $T_{4}, T_{5}$, $T_{6}, \alpha_{a v g}$ and $W_{f u l l}$, normalized by the forward link

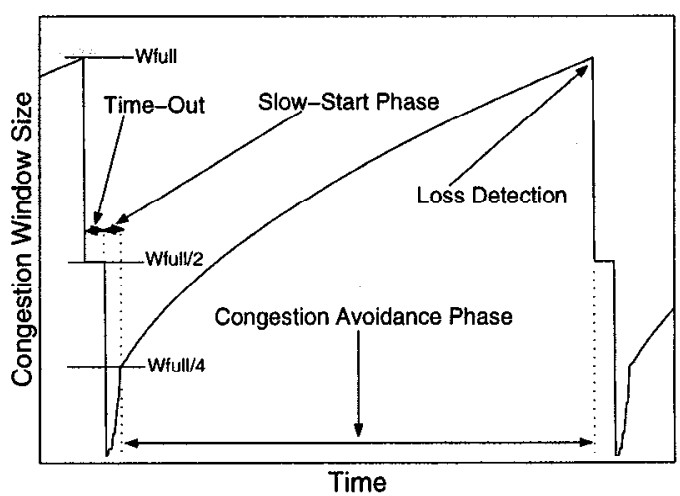

Fig. 4. Typical congestion window evolution for "busy connections" and $\alpha>1$.

capacity $\mu_{f}$ is given by:

$$
\gamma_{s a t}=\frac{\sum_{i=1}^{\left\lfloor\log _{2} \frac{\left.W_{\text {fult }}\right\rfloor}{4}\right\rfloor} 2^{i}+\sum_{i=1}^{\left\lfloor\frac{3 W_{\text {full }}}{4}\right\rfloor} \alpha_{\text {avg }}\left(\frac{W_{\text {full }}}{4}+i\right)}{\mu_{f} \tau_{\text {tot }}}
$$

where $\tau_{\text {tot }}$ is the total duration of a cycle given by:

$$
\tau_{\text {tot }}=T_{4}+\frac{3}{\mu_{r}}+R T O+\log _{2}\left(\frac{W_{\text {full }}}{4}\right) T_{5}+\frac{3 W_{\text {full }}}{4} \alpha_{\text {avg }} T_{6}
$$

$R T O$ is an estimate for the retransmission time-out and $\mu_{r}$ is the reverse link capacity.

\section{B. Simulation Results}

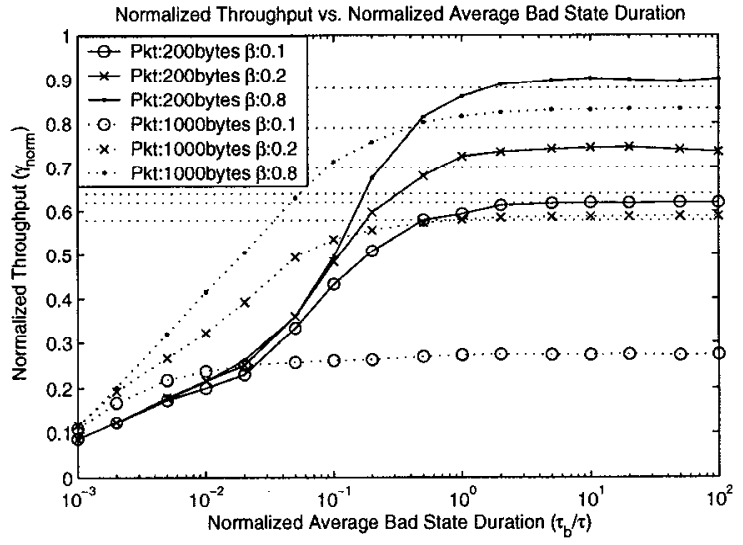

Fig. 5. Normalized throughput $\left(\gamma_{\text {norm }}\right)$ as a function of the normalized average bad state duration $\left(\tau_{b} / \tau\right)$ for $\rho=10^{-3}$. The horizontal dotted lines are results from equation (4).

Simulation traces were obtained using the same parameters as in Section $I V-B$ only now with $\alpha=3$ fixed (the forward bandwidth is also kept fixed at $1 \mathrm{Mbps}$ while $\mu_{f}$ and $\mu_{r}$ vary with the packet size). Results are 
shown in Fig.5. $\Lambda$ gain lossy connections are on the left end of the plot while busy connections are on the right. The performance sensitivity to packet sizes and buffer sizes remains as described in Section $I V-B$ only now asymmetry is responsible for an overall performance degradation. Among the six scenarios that were simulated, two of them do not satisfy $B_{f}>3 \alpha$. For 1000 bytes packet and $\beta=0.1\left(B_{f}<\alpha\right)$ throughput is significantly lower than the analytical results, while for 1000 bytes packet and $\beta=0.2\left(\alpha<B_{f}<3 \alpha\right)$ only slightly lower. Table II shows a comparison between analytical and simulation results for $\alpha \geq 1$. Fig.6

\section{TABLE II}

Throughput Saturation Limits for $\alpha>1$.

\begin{tabular}{|c|c|c|c|}
\hline \hline$\beta$ & Pkt Size & Eq. (2) & Simulation \\
\hline \hline \multirow{2}{*}{0.1} & 200 & 0.641 & 0.620 \\
\cline { 2 - 4 } & 1000 & 0.577 & 0.274 \\
\hline \hline \multirow{2}{*}{0.2} & 200 & 0.696 & 0.736 \\
\cline { 2 - 4 } & 1000 & 0.621 & 0.588 \\
\hline \hline \multirow{2}{*}{0.8} & 200 & 0.876 & 0.900 \\
\cline { 2 - 4 } & 1000 & 0.787 & 0.832 \\
\hline \hline
\end{tabular}

illustrates how larger propagation delays impact the results and yield a narrower performance difference between the the two choices of packet sizes in busy connections. Only results for $\beta=0.8$ are shown.

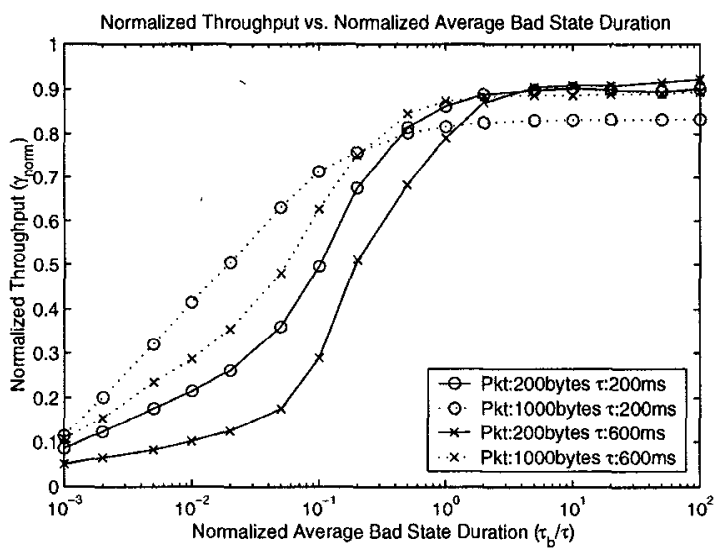

Fig. 6. Normalized throughput $\left(\gamma_{\text {norm }}\right)$ as a function of the normalized average bad state duration $\left(\tau_{b} / \tau\right)$ for different propagation delays and packet sizes $\left(\rho=10^{-3}\right)$.

\section{CONCLUSIONS}

This paper provides some insights on the effects of choosing packets sizes for a TCP-Reno connection over a channel with correlated errors. For the scenario where performance is dominated by congestion, analytical expressions were suggested and validated through simulations. Results show that performance sensitivity to the choice of packet size is reduced when the connection is dominated by congestion. In this case, connections using smaller packet sizes are able to achieve slightly higher throughput for both $\alpha \leq 1$ and $\alpha>1$. When link losses dominate the connection, sensitivity to packet size is higher and larger packets allow for best results.

\section{REFERENCES}

[1] T.V. Lakshman, U. Madhow and B. Suter, "Window-Based Error Recovery and Flow Control with Slow Acknowledgment Channel: A Study of TCP/IP Performance" - Proceedings of INFOCOM 1997, vol. 5, no. 3, pp. $336-350$, June 1997.

[2] V. Jacobson, "Compressing TCP/IP Headers for Low-Speed Serial Links" - IETF RFC1144, February, 1990.

[3] M. Allman, "Ongoing TCP Research Related to Satellites" - IETF RFC2760, February, 2000.

[4] M. Degermark, M. Engan, B. Nordgren, S. Pink, "Low-Loss TCP/IP Header Compression for Wireless Networks" - ACM - Baltzer Journal on Wireless Networks, vol.3, no.5, pp. 375$387,1997$.

[5] R. Braden, "Requirements for Internet Hosts - Communication Layers" - IETF RFC1122, October, 1998.

[6] I. T. Ming-Chit, D. Jinsong, W. Wang, "Improving TCP Performance Over Asymmetric Networks" - ACM SIGCOMM Communication Computer Review, vol. 30, No. 3, July 2000.

[7] M. Allman, "On the Generation and Use of TCP Acknowledgements" - ACM SIGCOMM Computer Communication Review, vol. 28, No. 5, October 1998.

[8] H. Balakrishnan, V. N. Padmanabhan, R. H. Katz, "The Effects of Asymmetry on TCP Performance" - ACM Mobile Networks and Applications (MONET) Journal, Vol. 4, No. 3, 1999.

[9] M. Albuquerque, "Effects of Asymmetry, Link Losses and Packet Sizing in the Performance of a TCP-Reno Connection" - MSEE Thesis - Department of Electrical Enogineering, University of Washington, March 2001.

[10] S. McCanne, S. Floyd, "The Network Simulator - ns-2" ns-2 Web Page (http://www.isi.edu/nsnam/ns/).

[11] M. Albuquerque, A. Abou-Zeid, S. Roy, "A Cautionary Note on Simulation Error Models for TCP Analysis: Discrete vs. Continuous Time" - PILC Mailing List (http://pilc.grc.nasa.gov/list/archive), November $21^{\text {st }} 2000$.

[12] M. Zorzi, A. Chockalingam, R. R. Rao, "Throughput Analysis of TCP on Channels with Memory" - IEEE Journal on Selected Areas in Communications, vol. 18, no. 7, pp. 1289 -1300 , July 2000.

[13] H. Balakrishnan, V. N. Padmanabhan, "TCP Performance Implications of Network Asymmetry" - IETF Internet Draft, September 1999.

[14] A. Abouzeid, S. Roy, M. Azizoglu, "Stochastic Modeling of TCP over Lossy Links" - Proceedings of INFOCOM 2000, Vol 3, pp. 1724 -1733, March 2000. 